\title{
PREVALENCE OF HYPERAMYLASAEMIA IN OPC POISONING AND ITS CLINICAL SIGNIFICANCE- A CROSS-SECTIONAL STUDY
}

\author{
Arcot Thanjan Maaran1, Kumanur Ethirajulu Govindarajulu2, Pillanallur Rajendran Saiprashanth³, Parthasarathy Prathiba ${ }^{4}$ \\ ${ }_{1}^{1}$ Assistant Professor, Department of General Medicine, Government Vellore Medical College and Hospital. \\ ${ }^{2}$ Associate Professor, Department of General Medicine, Government Vellore Medical College and Hospital. \\ 3 Postgraduate Student, Department of General Medicine, Government Vellore Medical College and Hospital. \\ ${ }_{4}^{4}$ Postgraduate Student, Department of General Medicine, Government Vellore Medical College and Hospital.
}

\section{BACKGROUND}

ABSTRACT

The incidence of organophosphorus poisoning has been steadily increasing and is typically endemic to developing countries like India, which has an economy based primarily on agriculture. The toxicity of organophosphorus compounds and the paucity of accessible medical facilities accounts for a high fatality rate. Their ease of access and several sociocultural factors play an important role in establishing organophosphorus compounds as a self-administered poison.

The objective of this study is to estimate the prevalence of hyperamylasaemia in OPC poisoning and to determine its clinical significance.

\section{MATERIALS AND METHODS}

This was a cross-sectional study conducted in the Department of General Medicine in Govt. Vellore Medical College for a period of 3 months starting from 01 June 2016 to 31 August 2016. All patients aged 13 and above getting admitted in medical wards and in Intensive Care Unit attached to Department of General Medicine, GVMCH, Vellore with documented history of OPC poison consumption or exposure were included in the study. Information were collected through a preformed and pre-tested proforma from each patient. Qualifying patients underwent detailed history, clinical, biochemical and radiological examination.

\section{RESULTS}

Among 75 patients with OPC poisoning 30 patients (40\%) had increased serum amylase levels, 14 (46.66\%) out of 30 patients with hyperamylasaemia patients had elevated serum lipase levels. Out of 14 patients 7 patients (9.33\%) had hyperlipasaemia, while 7 patients (9.33\%) among 14 patients with hyperlipasaemia were diagnosed with acute pancreatitis proven by biochemical and radiological investigations.

\section{CONCLUSION}

Hyperamylasaemia is frequently seen in organophosphate poisoning. In the study population, 7 patients were proved to have acute pancreatitis as a complication of OPC poisoning.

\section{KEYWORDS}

Organophosphate Poisoning, Acute Pancreatitis, Hyperamylasaemia, Hyperlipasaemia.

HOW TO CITE THIS ARTICLE: Maaran AT, Govindarajulu KE, Saiprashanth PR, et al. Prevalence of hyperamylasaemia in OPC poisoning and its clinical significance- a cross-sectional study. J. Evolution Med. Dent. Sci. 2017;6(91):6497-6500, DOI: $10.14260 /$ jemds $/ 2017 / 1411$

\section{BACKGROUND}

The incidence of organophosphorus poisoning has been steadily increasing and is typically endemic to developing countries like India, which has an economy based primarily on agriculture. The toxicity of organophosphorus compounds and the paucity of accessible medical facilities accounts for a high fatality rate. Their ease of access and several sociocultural factors play an important role in establishing organophosphorus compounds as a self-administered poison. The incidence is higher among young economically active group with fatality ratio of $20 \%$. In India, OP compounds

'Financial or Other Competing Interest': None.

Submission 03-10-2017, Peer Review 12-11-2017,

Acceptance 18-11-2017, Published 27-11-2017.

Corresponding Author:

Dr. Kumanur Ethirajulu Govindarajulu,

Associate Professor,

Department of General Medicine,

Government Vellore Medical College and Hospital,

Vellore.

E-mail: julugrh_sn@yahoo.co.in

DOI: $10.14260 /$ jemds/2017/1411

\section{(c) $($ ) $\odot$}

cause more self-poisoning deaths in southern and central India.

Organophosphorus insecticides include chlorpyrifos, phosphorotioic acid (Diazinon), dichlorvos, fenthion, parathion and malathion and sarin and carbamate insecticides include aldicarb, propoxur, carbaryl and bendiocarb.

The mechanism of action is irreversible inhibition of acetylcholinesterase causing accumulation of acetylcholine at muscarinic and nicotinic synapses and in CNS. There is also cholinergic stimulation of the pancreas and the Sphincter of Oddi (SO). In animal models, this results in both increased pancreatic secretion and increased SO activity. ${ }^{1}$ Studies have shown that excessive cholinergic stimulation using an acetylcholine agonist may lead to acute pancreatitis. In patients with OPC compound poisoning, there is an irreversible inhibition of cholinesterase resulting in delayed breakdown of synaptic acetylcholine leading to acute pancreatitis in humans. Case reports on clinical significance of hyperamylasaemia and acute pancreatitis following acute organophosphorus compound ingestion have been reported now and then. In our study, the prevalence of 
hyperamylasaemia and associated factors in patients with OPC poisoning in a tertiary care centre and its clinical significance will be reiterated, so that our study may serve as a harbinger of several larger serial studies.

\section{MATERIALS AND METHODS}

\section{Methodology}

This is a cross-sectional study conducted in Govt. Vellore Medical College for a duration of 3 months from 01 June 2016 to 31 August 2016.

\section{Sampling Method}

Convenience sampling technique was used.

\section{Inclusion Criteria}

All patients aged 13 and above getting admitted in medical wards and intensive care unit attached to Department of General Medicine, GVMCH with history of OPC poisoning either intentional or accidental were included in the study.

\section{Exclusion Criteria}

Whereas patients with history of exposure to an entirely different poison other than OP poison, patients with OP poisoning mixed with any other poison, chronic alcoholics, patients with history suggestive of gall stone disease, parotid gland disease, patients with history of lipid disorders, hyperparathyroidism, patients with history of renal or hepatic disease and intake of drugs likely to produce pancreatitis were excluded from this study.

A total of 75 such patients were selected for this study. Information were collected through a preformed and pretested proforma from each patient. Qualifying patients underwent detailed history, clinical, biochemical and radiological examination.

The composition of the poison was recorded from the label of the container. The reference serum amylase level was $0-140 \mathrm{U} / \mathrm{L}$ and the reference lipase level was $0-160 \mathrm{U} / \mathrm{L}$. Acute pancreatitis was diagnosed with characteristic symptoms, greater than three-fold elevation of serum amylase or lipase and characteristic radiological findings in USG abdomen or in CT abdomen.

\section{Statistical Analysis}

Data analysis was done using SPSS software V16. The prevalence is expressed in percentage. Quantitative data were expressed in mean, minimum, maximum and standard deviation. The qualitative data was expressed by Chi-square test. The difference was considered statistically significant if $p$ value was $\leq 0.05$.

\section{RESULTS}

\section{Distribution of OPC Poisoning in the Study Population}

Among 75 patients with OPC poisoning during the study period 70 patients had attempted suicide (93.33\%), 5 patients were exposed to OPC poisoning accidentally (6.66\%), 70 patients had exposure to OPC through ingestion of the poison (93.33\%), whereas 4 patients had exposure through inhalation $(5.33 \%)$ and 1 patient through cutaneous absorption (1.33\%).

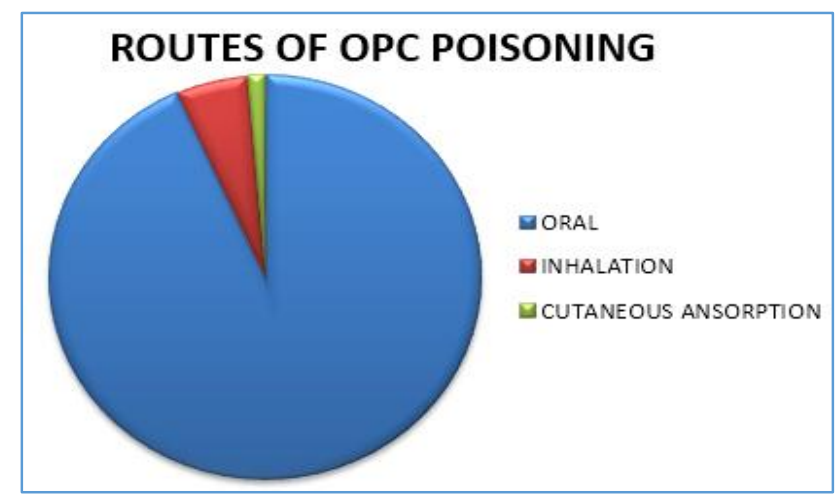

\section{Sex Distribution}

Sex distribution showed 42 males (56\%) and 33 females (44\%), male-to-female ratio was 1: 0.8 .

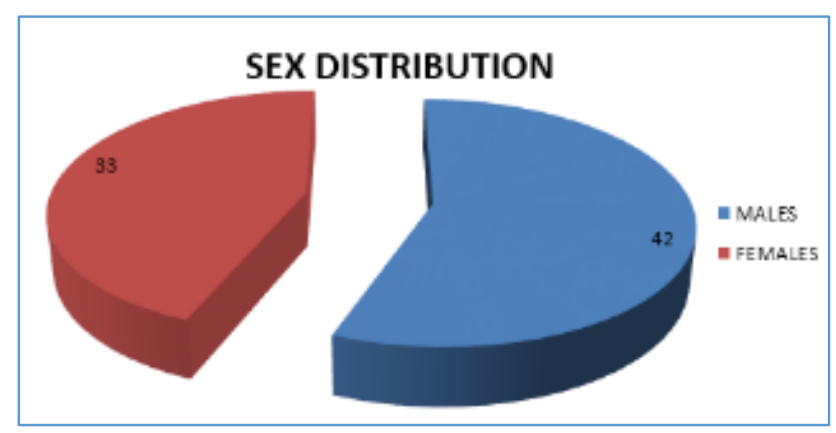

Mean age of presentation was $41 \pm 13.21$ years. The youngest was 14 years and the oldest was 70 years. The mean serum amylase level was $152.85 \pm 127.83 \mathrm{U} / \mathrm{L}$. The minimum and maximum values were 30 and 520 , respectively.

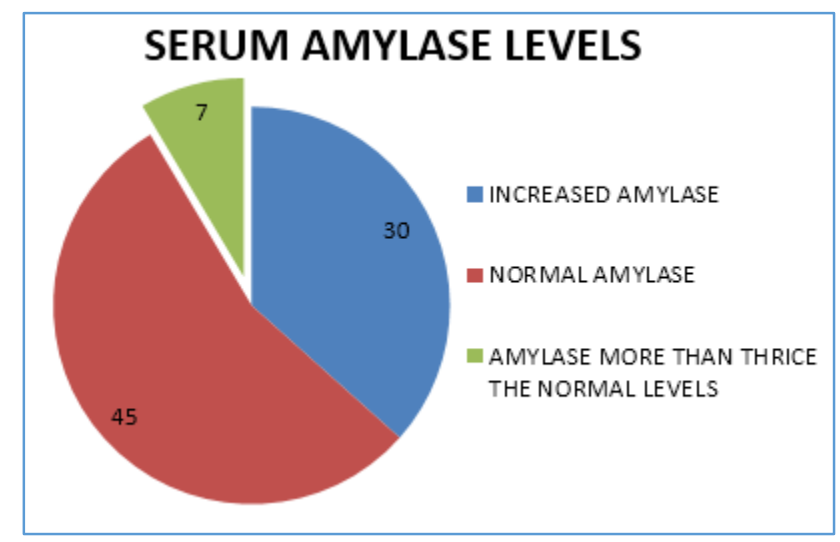

Throughout the study period 30 patients (40\%) had increased serum amylase levels, out of which 7 patients had amylase levels more than thrice the upper limit of normal serum amylase. Increased amylase levels were found more commonly in older age group ( $\mathrm{p}<0.00$ with 4 degrees of freedom). The mean lipase level was $141.69 \pm 139.22$ with a minimum of 30 and a maximum of $602 \mathrm{U} / \mathrm{L}$. The levels of serum lipase were found to be higher in older age group $(\mathrm{p}<$ 0.00 with 4 degrees of freedom). Sex was not found to play any role in serum lipase and serum amylase levels; 14 $(46.66 \%)$ out of 30 patients with hyperamylasaemia patients had elevated serum lipase levels, out of which 7 (50\%) patients had thrice the upper limit of serum lipase and 7 (50\%) patients had serum lipase values less than thrice the upper limit. Increased lipase levels were associated with 
increased amylase levels $(p<0.00$ with 4 degrees of freedom).

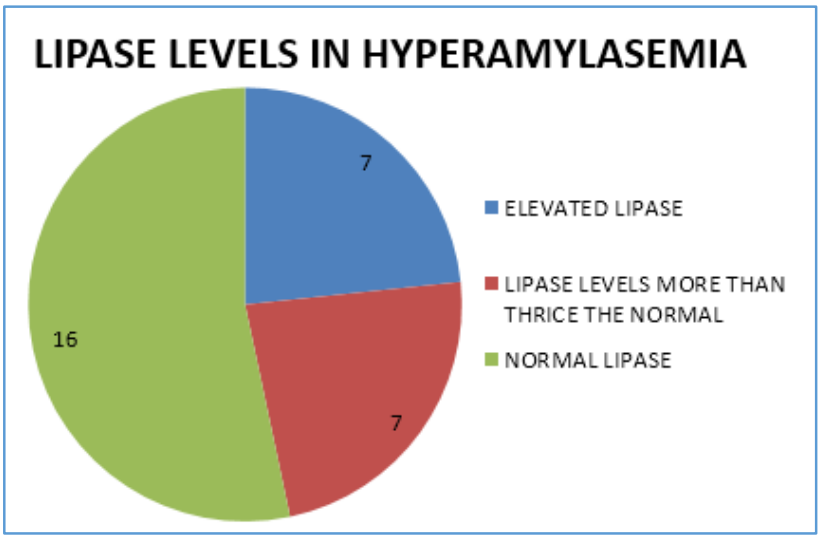

Among 75 patients 30 patients with hyperamylasaemia has leucocytosis, transaminitis, elevated LDH and CPK levels. In patients with normal serum amylase levels, leucocytosis was observed in $37.5 \%$ of patients, raised LDH was found in 6 patients $(12.5 \%)$, raised CPK was seen in $2(4.16 \%)$ patients and raised SGPT was seen in 2 patients (4.16\%).

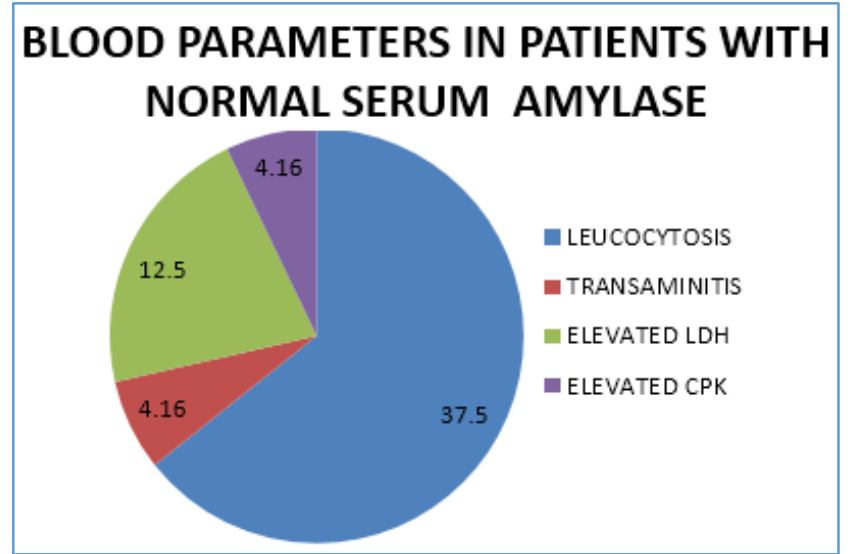

Among 75 patients 29 patients (38.6\%) reported with excessive salivation, while diarrhoea was seen in 26 (34.6\%) of patients and vomiting in $2 \%$ of patients. Abdominal pain was seen in 18 patients (24\%), out of these $13(72.2 \%)$ had hyperamylasaemia and 7 patients $(9.33 \%)$ among them were diagnosed with acute pancreatitis proven by Biochemical and Radiological investigations. The prevalence of pancreatitis in patients with hyperamylasaemia and hyperlipasaemia were statistically significant ( $p<0.00$ with 2 degrees of freedom). Pancreatitis was more common in older age groups $(\mathrm{p}<0.00$ with 2 degrees of freedom). Out of 75 patients 3 patients died, all of them due to respiratory failure.

\section{DISCUSSION}

OP compound poisoning now almost unheard of in the western world is still now rampant in developing nations, particularly in south Asian countries. This is due to the fact that agriculture has been the backbone of south Asian economies and OPCs are effective pesticides and are easily available despite strict regulations. OP poisoning has a very low mortality rate if effectively managed. Several studies have documented pancreatitis as an important complication in patients with OP poisoning, ${ }^{2}$ but there is no definitive marker for diagnosing OPC-induced acute pancreatitis.
Establishing a valid marker will dramatically benefit the clinician in planning further course of treatment and thereby directly reducing the mortality rate. In Veterans General Hospital, National Yang-Ming University, a retrospective study was done based on medical records of 121 OPC poisoning patients by Wui-Chiang Lee et $\mathrm{al}^{3}{ }^{3}$ over a course of three years. Various biomarkers of pancreatitis, serum cholinesterase and clinical manifestations were all analysed. About 44 patients (36\%) had serum amylase levels greater than $360 \mathrm{U} / \mathrm{L} ; 28$ patients with hyperamylasaemia was tested for lipase in serum, out of which 9 patients had serum lipase levels greater than $380 \mathrm{U} / \mathrm{L}$. The study concluded that increased serum amylase levels is a frequent finding in patients with OPC poisoning and serum lipase not amylase was found to be a sensitive marker for diagnosing acute pancreatitis in OPC poisoning. Hence, serum lipase level is indicated in patients with increased amylase levels in patients with OP poisoning.

A retrospective study was done in 1996 by N Matsumiya et $\mathrm{al}^{4}$ in Kyodo General Hospital, Ibaraki, Japan in an ICU setup. The study was done to analyse the incidence of respiratory failure in OPC poisoning; 32 OPC poisoning patient records were selected, 16 patients among them had developed respiratory failure and had received ventilator support. Hyperamylasaemia was found in patients who had developed respiratory failure. The study concluded that the hyperamylasaemia in OPC poisoning can be used as a predictive marker for respiratory failure. A prospective study was conducted in a North Indian tertiary care setup by Singh $\mathrm{S}$ and Bhardwaj $\mathrm{U}$ et $\mathrm{al}^{5}$ between June 2001 - June 2005, to determine the incidence of hyperamylasaemia and acute pancreatitis in patients with OPC compound poisoning; 79 patients were studied, out of which 37 patients $(46.95 \%)$ demonstrated hyperamylasaemia (> $200 \mathrm{SU}$ ). Among them 3 patients had levels greater than 300 SU. One patient had bulky pancreas in USG study confirmed by CT abdomen. Two other patients did not demonstrate significant radiological features of pancreatitis. The study showed that whereas increased serum amylase is a common finding, acute pancreatitis as a complication of OPC compound poisoning is very rare. A similar prospective study by I Sahin et al was conducted in a tertiary care centre in Turkey in $2002^{6}$ to determine the prevalence of pancreatitis in patients with OPC poisoning. A total of 47 patients who had OPC poisoning were studied; 4 out of 47 patients had significantly elevated serum amylase levels (> $300 \mathrm{U} / \mathrm{L}$ ) as well as lipase levels (> $60 \mathrm{U} / \mathrm{L}$ ) Two patients who had serum amylase levels between 100 . $300 \mathrm{U} / \mathrm{L}$ had significantly elevated lipase levels. Among the patients with normal serum amylase levels, none had elevated lipase levels. In the study, a total of $12.76 \%$ were diagnosed with acute pancreatitis. The study shows the importance of routinely performing serum amylase and serum lipase levels in patients with OPC poisoning for earlier detection of acute pancreatitis, thereby initiating early treatment. A descriptive study was done in Pakistan by Ahmed Arshia et $\mathrm{al}^{7}$ in the Medicine Department at Abbasi Shaheed Hospital over a period of six months from 16th June 2006 to December 2006. Over 90 patients were included in the study among which 28 had hyperamylasaemia, 9 had hyperlipasaemia and 2 had evidence of pancreatitis.

In our study, we had a fraction of OPC poisoning patients whose serum amylase levels were comparable with the 
International studies and the percentage of patients with acute pancreatitis is significantly higher compared to all above mentioned studies, albeit the study by I Sahin et al had demonstrated a higher fraction.

Limitations of our study is that the marker levels were all done within 24 hours of admission and the upper limits of the biomarker levels were rather stringent in nature when compared to other studies. A more dynamic study is needed to establish the purportedly claimed correlation by serial monitoring of blood levels of all the markers to look for any rising trends in levels that may mark the onset of pancreatitis. Owing to the lack of facilities to measure serum cholinesterase levels in our labs, this study was unable to establish such a correlation. Serum cholinesterase should be measured along with markers to look for association in future studies.

\section{CONCLUSION}

Acute pancreatitis in OPC poisoning is not uncommon. Measuring serum amylase in patients with OPC poisoning is the first step leading to a diagnosis of acute pancreatitis in such patients. This study aims to enlighten the clinician regarding importance of measuring serum amylase levels in OPC poisoning, which may become life saving for many patients.

\section{REFERENCES}

[1] Chen JW, Saccone GT, Toouli J. Sphincter of Oddi dysfunction and acute pancreatitis. Gut 1998;43(3):305-8.

[2] Greenberger NJ, Toskas PP, Isselbacher KJ. Acute and chronic pancreatitis. In: Fauci SA, Braunwald E, Isselbacher $\mathrm{KJ}$, et al. eds. Harrison's principles of internal medicine. 14th edn. New York: McGraw-Hill 1998:1741-52.

[3] Lee WC, Yang CC, Deng JF, et al. The clinical significance of hyperamylasemia in organophosphate poisoning. Journal of Toxicology Clinical Toxicology 1998;36(7):673-81.

[4] Matsumiya N, Tanaka M, Iwai M, et al. Elevated amylase is related to the development of respiratory failure in organophosphate poisoning. Human \& Experimental Toxicology 1996;15(3):250-3.

[5] Singh S, Bhardwaj U, Verma SK, et al. Hyperamylasemia and acute pancreatitis following anticholinesterase poisoning. Human \& Experimental Toxicology 2007;26(6):467-71.

[6] Sahin IC, Onbasi K, Sahin H, et al. The prevalence of pancreatitis in organophosphate poisonings. Human \& Experimental Toxicology 2002;21(4):175-7.

[7] Ahmed A, Begum I, Aquil N, et al. Hyperamylasemia and acute pancreatitis following organophosphate poisoning. Pak J Med Sci 2009;25(6):957-61. 